\title{
Spectroscopic Characterization of Soybean Lipoxygenase-1 Mutants: the Role of Second Coordination Sphere Residues in the Regulation of Enzyme Activity ${ }^{\dagger}$
}

\author{
Gerhard Schenk, ${ }^{\ddagger}$ Michael L. Neidig, ${ }^{\ddagger}$ Jing Zhou, ${ }^{\ddagger}$ Theodore R. Holman,*, and Edward I. Solomon*, \\ Department of Chemistry, Stanford University, Stanford, California 94305-5080, and Department of Chemistry \\ and Biochemistry, University of California, Santa Cruz, California 95064
}

Received December 19, 2002; Revised Manuscript Received April 14, 2003

\begin{abstract}
Lipoxygenases are non-heme iron enzymes, which catalyze the stereo- and regiospecific hydroperoxidation of unsaturated fatty acids. Spectroscopic studies on soybean lipoxygenase have shown that the ferrous form of the enzyme is a mixture of five- and six-coordinate species (40 and $60 \%$, respectively). Addition of substrate leads to a purely six-coordinate form. A series of mutations in the second coordination sphere (Q697E, Q697N, Q495A, and Q495E) were generated, and the structures of the mutants were solved by crystallography [Tomchick et al. (2001) Biochemistry 40, 7509-7517]. While this study clearly showed the contribution of H-bond interactions between the first and the second coordination spheres in catalysis, no correlation with the coordination environment of the $\mathrm{Fe}^{\mathrm{II}}$ was observed. A recent study using density-functional theory [Lehnert and Solomon (2002) J. Biol. Inorg. Chem. 8, 294-305] indicated that coordination flexibility, involving the Asn694 ligand, is regulated via H-bond interactions. In this paper, we investigate the solution structures of the second coordination sphere mutants using $\mathrm{CD}$ and MCD spectroscopy since these techniques are more sensitive indicators of the first coordination sphere ligation of $\mathrm{Fe}^{\mathrm{II}}$ systems. Our data demonstrate that the iron coordination environment directly relates to activity, with the mutations that have the ability to form a five-coordinate/six-coordinate mixture being more active. We propose that the H-bond between the weak Asn694 ligand and the Gln697 plays a key role in the modulation of the coordination flexibility of Asn694, and thus, is crucial for the regulation of enzyme reactivity.
\end{abstract}

Lipoxygenases (LOs) $)^{1}$ are mononuclear non-heme iron dioxygenases that catalyze the stereo- and regiospecific hydroperoxidations of $c i s, c i s-1,4$ polyunsaturated pentadienyl containing fatty acids. LOs have been isolated and characterized from numerous mammalian and plant sources and are believed to occur ubiquitously throughout these two phyla $(1-3)$. In mammalian organisms, there are multiple LOs with differing regio- and stereospecificity against their substrate, arachidonic acid (AA). The products of these reactions are further metabolized by an array of enzymes leading to the formation of two major classes of cell effector molecules,

$\dagger$ This research was supported by the National Institutes of Health (GM-40392 to E.I.S. and GM- 56062-06 to T.R.H.) and a National Science Foundation Graduate Fellowship to M.L.N.

* To whom correspondence should be addressed. (E.I.S.) Telephone: (650) 723-9104. Fax: (650) 725-0259. E-mail: edward.solomon@stanford.edu. (T.R.H.) Telephone: (831) 459-5884. Fax: (831) 459-2935. E-mail: tholman@chemistry.ucsc.edu.

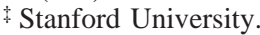

$\S$ University of California, Santa Cruz.

${ }^{1}$ Abbreviations: LO, lipoxygenase; wt, wild-type; sLO-1, soybean lipoxygenase-1; Q495E, Gln495Glu sLO-1; Q495A, Gln495Ala sLO-1; Q495N, Gln495Asn sLO-1; Q697N, Gln697Asn sLO-1; Q697E, Gln697Glu sLO-1; 15-hLO, human reticulocyte 15-lipoxygenase; 15rLO, rabbit reticulocyte 15-lipoxygenase; LA, linoleic acid; AA, arachidonic acid; OA, oleic acid; 13-HPOD, 13-hydroperoxy-9,11-(Z,E)octadecadienoic acid; $5 \mathrm{C}$, five coordinate; $6 \mathrm{C}$, six coordinate; DFT, density-field theory; ICP-MS, inductively coupled plasma mass spectroscopy; $\mathrm{CD}$, circular dichroism; $\mathrm{MCD}$, magnetic circular dichroism; VTVH, variable temperature, variable field; EXAFS, extended X-ray absorption fine structure; EPR, electron paramagnetic resonance. leukotrienes and lipoxins, which are mediators in anaphylactic and inflammatory disorders $(4,5)$. Additionally, LO products are involved in a variety of metabolic functions, including organelle degradation $(6)$, transcription regulation (7), and possibly tumor cell metastasis (8). For plant LOs, the main substrates are linoleic acid (LA) and linolenic acid, which are further metabolized to plant hormones, such as jasmonic acid. These hormones are involved in a variety of metabolic functions including plant growth and development, senescence, and cellular response to wounding and infections by pathogens (1). Soybeans have three LO isoforms (sLO-1, sLO-2, and sLO-3), which have homologous sequences and molecular weights of $\sim 96 \mathrm{kDa}$ (9). This study focuses on sLO-1 because of its stability and the fact that most kinetic, structural and spectroscopic studies have involved this isoform.

The mechanism of sLO-1 has been studied by a wide range of kinetic and spectroscopic methods (for a review see ref 3 ). At least $90 \%$ of the isolated enzyme is in its inactive, ferrous form (10), which does not react with substrate and dioxygen (11). Addition of 1 equiv of the reaction product, 13-hydroperoxy-9,11-(Z,E)-octadecadienoic acid (13-HPOD), oxidizes the $\mathrm{Fe}^{\mathrm{II}}$, thus activating the enzyme (12). The generally accepted mechanism of catalysis invokes a $\mathrm{H}$-atom abstraction from the $\mathrm{C} 11$ position of the substrate by an $\mathrm{Fe}^{\mathrm{III}}$ $\mathrm{OH}(13,14)$, accompanied by the generation of an $\mathrm{Fe}^{\mathrm{II}}-\mathrm{OH}_{2}$ species and a substrate radical $(15,16)$. The $\mathrm{H}$-atom abstraction is one of three rate-limiting steps at room 
temperature and is associated with a large, primary kinetic isotope effect of $\sim 80$ (17). This observation has led to the conclusion that the hydrogen is transferred from the substrate to the active site by a tunneling mechanism. A recent DFT study implicated that this $\mathrm{H}$-atom transfer step corresponds to a proton-coupled electron transfer (PCET), where the electron tunnels directly from the substrate to the ferric ion (18). The substrate radical, formed after the PCET, reacts with $\mathrm{O}_{2}$ to form a peroxyl radical, as evidenced by EPR spectroscopy (19-21). Subsequently, the $\mathrm{Fe}^{\mathrm{II}}-\mathrm{OH}_{2}$ is oxidized by the peroxyl radical, although the mechanism remains unclear. It has been proposed that a rearrangement in the active site could lead to the formation of a purple species, where the peroxo group is directly linked to the iron $(22$, 23). However, it is uncertain if this species is a reaction intermediate or a dead-end product since the peroxide is attached to the opposite side of the abstracted hydrogen and thus requires a major reorientation of the intermediate. The catalytic cycle is completed by product release, and the active $\mathrm{Fe}^{\mathrm{III}}-\mathrm{OH}$ enters the next reaction cycle.

Crystal structures for ferrous LOs have been solved for two soybean isoforms (sLO-1 and sLO-3) $(14,24-26)$ and rabbit reticulocyte $15-\mathrm{LO}$ (15-rLO) (27). The three structures display a similar overall folding pattern. The main difference in their ferrous active sites is the replacement of a histidine ligand in 15-rLO by an asparagine in the two sLOs. The rhombically distorted coordination sphere is completed by three histidines, one mono-dentate carboxylic acid (the C-terminal isoleucine), and a water ligand. In sLOs, the asparagine ligand is at least $2.9 \AA$ away from the metal ion, indicating a very weak bonding interaction. Furthermore, the $\mathrm{Fe}^{\mathrm{II}}-$ Asn distance in sLO- 1 varies over $\approx 0.5 \AA$, depending on the crystallization conditions of the particular structure $(14,24,25)$. A combination of circular dichroism (CD) and magnetic CD (MCD) spectroscopies has provided insight into the nature of the $\mathrm{Fe}^{\mathrm{II}}-$ Asn interaction in solution $(3,28)$. In the absence of substrates, the coordination number reflects an equilibrium between five- and six-coordinate species $(5 \mathrm{C} /$ 6C $\sim 40: 60 \%$ ). Addition of anaerobic substrate and/or small organic molecules, such as ethanol and glycerol, shifts this equilibrium to a full conversion to the $6 \mathrm{C}$ form. In contrast, the ferrous active site of mammalian 15-LOs is always 6C, independent of the presence of substrate (28), because the active site contains a relatively strong histidine donor ligand instead of the weaker asparagine ligand present in sLOs. This histidine is anticipated to form a stronger bond with $\mathrm{Fe}^{\mathrm{II}}$ since it is a more covalent ligand than the weak field asparagine, which is clearly seen in the crystal structure of 15-rLO (27). These combined spectroscopic and crystallographic data have provided a clear indication of coordination flexibility in ferrous sLOs, which is ascribed to the presence of a weak asparagine ligand (Asn694 in sLO-1).

The ferric form of mammalian LOs has a rhombic ZFS in EPR and is 6C (29-33). Initial studies using iron K-edge X-ray absorption spectroscopy (13) suggested a similar coordination sphere in sLO-1. However, EPR spectroscopy accompanied by a ligand field analysis of the axial ZFS of a non-heme $\mathrm{Fe}^{\mathrm{III}}$ complex indicated that the ferric form of sLO-1 is best described as 5C (33). Addition of glycerol or substrate does not alter the EPR spectra (i.e., coordination number) for either sLO-1 or mammalian LOs. Since the $\mathrm{H}$-atom abstraction and concomitant reduction of $\mathrm{Fe}^{\mathrm{III}}$ are crucial steps in the LO reaction mechanism (3), the $\mathrm{p} K_{\mathrm{a}}$ of the water ligand of the $\mathrm{Fe}^{\mathrm{II}}$ and the reduction potential of the metal ion are important factors in determining enzyme reactivity (34). Therefore, the observation of a $6 \mathrm{C}$ ferrous and a $5 \mathrm{C}$ ferric form in sLOs has been interpreted in terms of an optimization of the catalytic efficiency of these enzymes (3). A 5C site destabilizes the ferric state, thus increasing the reduction potential, while a $6 \mathrm{C}$ ferrous site ensures a high $\mathrm{p} K_{\mathrm{a}}$ of the bound water. This model suggests that the observed coordination flexibility in sLOs is important in regulating enzyme activity and predicts that mammalian 15 -LOs are less reactive since they remain $6 \mathrm{C}$ in both the ferric and the ferrous state. Indeed, the $k_{\text {cat }}$ measured for the human 15-LO (15-hLO; $\left.6 \mathrm{~s}^{-1}\right)(35)$ is much smaller than that determined for sLO-1 $\left(280 \mathrm{~s}^{-1}\right)$ (17). This model is further supported by a site-directed mutagenesis study where the weak asparagine ligand in sLO-1 was replaced by a histidine, N694H, rendering the sLO-1 coordination environment equivalent to that of $15-\mathrm{hLO}$ and $15-\mathrm{rLO}$ (36). As expected, $\mathrm{N} 694 \mathrm{H}$ remains $6 \mathrm{C}$, independent of oxidation state or the presence of inhibitors or small organic molecules, and its reduction potential is lowered relative to sLO-1. The kinetic parameters of $\mathrm{N} 694 \mathrm{H}$ were reduced dramatically, similar to those of 15 -hLO $\left(k_{\mathrm{cat}}=10 \mathrm{~s}^{-1}\right)$ and corroborate our hypothesis that the $5 \mathrm{C} / 6 \mathrm{C}$ flexibility of the ferrous sLO-1 is important to the reactivity of the ferric state.

From the foregoing discussion, the coordination flexibility in sLOs plays an important role in the regulation of enzyme activity. An examination of the crystal structure of wildtype (wt) sLO-1 (14) indicates the presence of an extensive hydrogen bonding network that connects the weakly bound asparagine ligand (Asn694) via the two second coordination sphere residues, Gln697 and Gln495, to an equatorial iron ligand, His499 (Figure 1). A similar network is also present in 15-rLO (27), and it was speculated that interactions between the substrate and this hydrogen bond network are responsible for the observed sensitivity of the coordination number in ferrous sLOs (14). Previously, Gln697 and Gln495 were mutated (Q697E, Q697N, Q495E, and Q495A) (Figure $1)$, and their kinetic properties were related to their crystal structures (14). This mutant data provided evidence that structural changes of the hydrogen bond network could account for the observed kinetic differences. However, the measured $\mathrm{Fe}^{\mathrm{II}}-\mathrm{Asn} 694$ distances in the crystal structures could not be directly related to enzyme activity. A recent DFT study, however, indicated that the protein could control the $\mathrm{Fe}^{\mathrm{II}}-\mathrm{Asn}$ distance by adjusting the spatial orientation of this ligand (18). As mentioned above, for wt sLO-1, X-ray crystallography may not accurately estimate specific ligandmetal bonding interactions in solution because of disorder or effects of the crystallization process. However, the ligand field at the $\mathrm{Fe}^{\mathrm{II}}$ center is extremely sensitive to specific bonding interactions and can be directly probed by CD and MCD spectroscopy. In the current paper, we utilize CD and MCD spectroscopy to gain insight into both the geometric and the electronic structure of the $\mathrm{Fe}^{\mathrm{II}}$ site in these sLO-1 mutants and correlate these data to the regulation of enzyme activity. This study employs a combination of structural and spectroscopic techniques to obtain detailed insight into the reaction mechanism. 


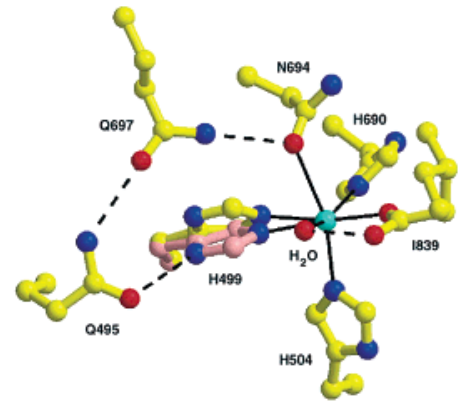

wt sLO-1

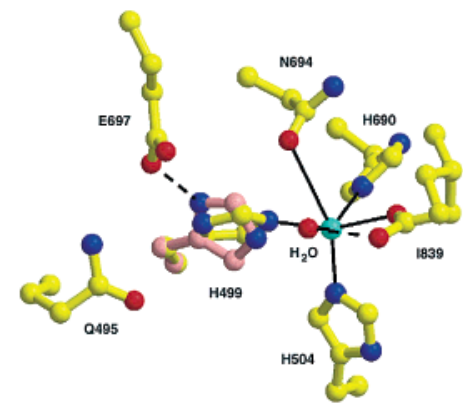

Q697E sLO-1

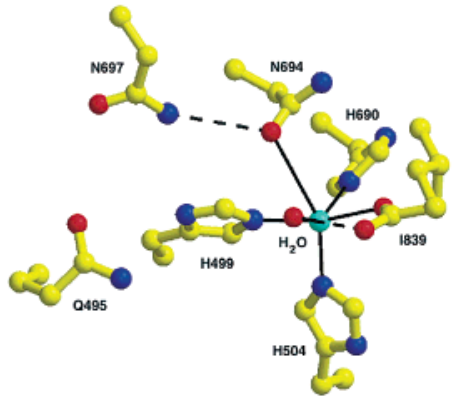

Q697N sLO-1
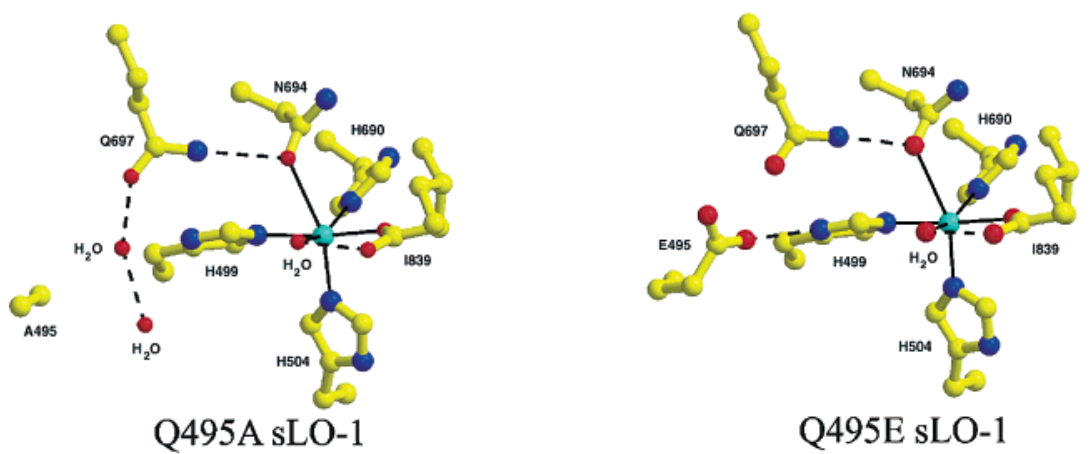

FIGURE 1: Active site geometry of wt sLO-1 and four second coordination sphere mutants. Bonds between the iron and its ligands are drawn as solid lines; H-bond interactions are drawn as dashed lines. Oxygen atoms are drawn in red, nitrogens in blue, carbons in yellow, and the iron atom in cyan. Possible alternate conformations of His499 are shown in pink.

\section{MATERIALS AND METHODS}

Materials. Oleic acid (OA) was obtained from Aldrich Chemical Co., and molecular biology reagents were purchased from New England Biolabs (Beverly, MA). Buffers were made in $\mathrm{D}_{2} \mathrm{O}$ (Sigma). All reagents used were reagent grade or better and were used without further purification.

Mutagenesis, Expression, and Protein Purification. Sitedirected mutagenesis, overexpression, and purification of wt and mutant sLO-1 enzymes followed a protocol outlined previously $(14,36)$. In brief, following expression of the protein in BL21 (Escherichia coli) the cells were harvested by centrifugation, and their membranes were disrupted by sonication. Cell debris was pelleted, and the supernatant was first dialyzed against $20 \mathrm{mM}$ Bis-Tris buffer ( $\mathrm{pH}$ 6.0) and then applied to a SP-Sephadex high-flow ion exchange column (Pharmacia), which was equilibrated with the same buffer. Eluted fractions containing lipoxygenase activity were pooled, concentrated, and dialyzed again against $20 \mathrm{mM}$ BisTris buffer (pH 6.0) and applied to a Bio-S 20 ion exchange column (Bio-Rad). After buffer exchange into $50 \mathrm{mM}$ deuterated CHES ( $\mathrm{pD} 9.0$ ), the isolated enzyme was estimated to be at least 95\% pure (SDS-PAGE analysis).

Enzyme Activity and Iron Content. Lipoxygenase activity was determined by monitoring the absorption of the formation of product during the steady-state reaction at $234 \mathrm{~nm}$ $\left(\epsilon_{234}=25000 \mathrm{M}^{-1} \mathrm{~cm}^{-1}\right)$. The iron content was measured with a Finnegan ICP-MS apparatus, using standardized Fe solutions for calibration.

Near-IR/UV CD and MCD Spectroscopy. Spectra were recorded on a Jasco J200D spectropolarimeter (600-2100 $\mathrm{nm}$ ) with an $\mathrm{N}_{2}$-cooled $\mathrm{InSb}$ detector. Depolarization was estimated to be less than 5\% from the CD spectra of a nickel$(+)$-tartrate solution placed before and after the samples. For
$\mathrm{CD}$ at $5{ }^{\circ} \mathrm{C}$, a $0.5 \mathrm{~cm}$ path length infrasil quartz cuvette (Wilmad) was used. The spectra were corrected for buffer and buffer background. MCD samples were obtained from the $\mathrm{CD}$ samples by adding sucrose to supersaturation $(\sim 175$ $\mathrm{mg} / 100 \mu \mathrm{L}$ ). Glycerol- $d_{3}$ was not suitable as a glassing agent since its addition to both wt and mutant sLO- 1 affects their CD spectra, as observed previously (28). The MCD sample holder consists of two infrasil quartz disks separated by a $0.3 \mathrm{~cm}$ thick rubber O-ring. An approximately $120 \mu \mathrm{L}$ sample was filled into the gap between the two disks. Upon freezing in liquid $\mathrm{N}_{2}$, the sample was placed into the magnet (an Oxford Instruments SM4000-7T superconducting magnet). MCD spectra were measured at $1.6 \mathrm{~K}$ at fields up to $7 \mathrm{~T}$ and corrected for natural $\mathrm{CD}$ (at $0 \mathrm{~T}$ ) and baseline effects.

\section{RESULTS}

A high spin ferrous ion has a ${ }^{5} \mathrm{D}$ ground state, which splits into a triply degenerate ${ }^{5} \mathrm{~T}_{2 \mathrm{~g}}$ ground state and a doubly degenerate ${ }^{5} \mathrm{E}_{\mathrm{g}}$ excited state in an octahedral ligand field (37, 38). Biologically relevant $\mathrm{N}$ and $\mathrm{O}$ ligands (i.e., amino acids) lead to an energy separation of $\sim 10000 \mathrm{~cm}^{-1}$. Distortions from octahedral symmetry remove the degeneracies in both the ground and the excited states. CD and MCD provide a measure for the excited state splitting, whereas VTVH MCD can be used to probe the ground state splitting. In the present study, we are mainly concerned with excited-state information. The fundamental principles of the methodology applied have been discussed in detail elsewhere $(39,40)$. In brief, a distortion of the octahedral ligand arrangement by the low symmetry protein site generates a splitting of the ${ }^{5} \mathrm{E}_{\mathrm{g}}$ excited state by $\sim 2000 \mathrm{~cm}^{-1}$, centered around $10000 \mathrm{~cm}^{-1}$. Removal of an axial ligand leads to a square pyramidal $5 \mathrm{C}$ geometry, which characteristically splits the ${ }^{5} \mathrm{E}_{\mathrm{g}}$ state by $\geq 5000 \mathrm{~cm}^{-1}$, with transitions around 5000 and $\geq 10000 \mathrm{~cm}^{-1}$. Further 
Table 1: Gaussian Resolved Transitions in wt and Mutant sLO-1 in Absence and Presence of OA

\begin{tabular}{|c|c|c|c|c|c|c|}
\hline & \multicolumn{3}{|c|}{ Five-coordinate } & \multicolumn{3}{|c|}{ Six-coordinate } \\
\hline & $\begin{array}{l}\text { band } 1 \\
\left(\mathrm{~cm}^{-1}\right)\end{array}$ & $\begin{array}{l}\text { band } 2 \\
\left(\mathrm{~cm}^{-1}\right)\end{array}$ & $\begin{array}{c}\Delta \mathrm{E}_{\mathrm{g}} \\
\left(\mathrm{cm}^{-1}\right)\end{array}$ & $\begin{array}{l}\text { band } 1 \\
\left(\mathrm{~cm}^{-1}\right)\end{array}$ & $\begin{array}{l}\text { band } 2 \\
\left(\mathrm{~cm}^{-1}\right)\end{array}$ & $\underset{\left(\mathrm{cm}^{-1}\right)}{\Delta \mathrm{E}_{\mathrm{g}}}$ \\
\hline wild-type $^{a}$ & $<6000$ & 10670 & $>4700$ & 8630 & 10330 & 1700 \\
\hline Q697 & not present & not present & & & & \\
\hline $\mathrm{Q} 697 \mathrm{~N}^{a}$ & $<6000$ & 11500 & $>5500$ & 8650 & & 1350 \\
\hline & $<6000$ & & $>5$ & 510 & & 2030 \\
\hline & $<6000$ & 12220 & $>6200$ & 8380 & & 2440 \\
\hline ild-type ${ }^{b}$ & not present & not present & & 8600 & 10300 & 1700 \\
\hline & not present & not $\mathrm{p}$ & & 8070 & & 2390 \\
\hline & $<6000$ & 11400 & $>5400$ & 8400 & & 1720 \\
\hline Q495 & $<600$ & 1157 & $>5500$ & 40 & & 1910 \\
\hline $\mathrm{Q} 495 \mathrm{E}^{b}$ & not present & not present & & 8430 & 10760 & 2330 \\
\hline
\end{tabular}

${ }^{a}$ Resting enzyme. ${ }^{b}$ Resting enzyme plus OA.

alterations of the geometry and coordination number will lead to different energy splittings, as predicted by ligand field theory $(37,39,41)$ and observed experimentally in structurally defined mononuclear non-heme ferrous model complexes (42). Hence, CD and MCD can be used directly to probe the geometric environment of a ferrous center.

Previously, we applied this methodology to the study of wt sLO-1 (28) and showed that the spectrum of wt sLO-1 had three transitions at $<6000,8600$, and $10700 \mathrm{~cm}^{-1}$. The addition of glycerol leads to a significant change, displaying only two transitions, at 8600 and $10300 \mathrm{~cm}^{-1}$. From these observations, it was concluded that (i) glycerol is not a suitable glassing agent for MCD studies of SLO-1 and (ii) that the resting, ferrous enzyme is a mixture of six- and fivecoordinate species (28). Similar to glycerol, the presence of small organic molecules (ethanol or ethylene glycol), and more importantly, the anaerobic addition of substrate leads to a conversion of the $5 \mathrm{C} / 6 \mathrm{C}$ mixture to a purely $6 \mathrm{C}$ species, which is thought to be the catalytically relevant form. In contrast, addition of sucrose, another commonly used glassing agent, does not perturb the CD spectrum of wt sLO-1. The $5 \mathrm{C} / 6 \mathrm{C}$ mixture of wt-sLO-1 and its conversion to $6 \mathrm{C}$ were unaffected by $\mathrm{pH}$ (range, 5.6-11) or temperature, as seen by the similarity of the CD (276 K) and MCD (4.2 K) data (28).

Oleic acid (OA) is known to be an sLO inhibitor (43), which manifests a hyperbolic mixed-type inhibition, with OA binding to both the catalytic $\left(K_{\mathrm{I}} \approx 20 \mu \mathrm{M}\right)$ and the allosteric binding site $\left(K_{\mathrm{D}} \approx 36 \mu \mathrm{M}\right)(44)$. Here, we have carried out a $\mathrm{CD}$ titration to investigate the binding of $\mathrm{OA}$ to the active site of sLO-1. Incremental addition of OA to $1.3 \mathrm{mM}$ of sLO-1 leads to a conversion of sLO-1 from a mixture of $5 \mathrm{C}$ and $6 \mathrm{C}$ species to a purely $6 \mathrm{C}$ form. At 5 equiv excess of $\mathrm{OA}$, no further changes are observed (data not shown); the resulting spectrum (Figure 2) is indistinguishable from that of sLO-1 with added substrate, anaerobic linoleic acid, or glycerol. Because of the high concentration of sLO-1 and the tight binding of $\mathrm{OA}$, a saturation curve is not observable; therefore, the $K_{\mathrm{D}}$ can only be estimated to have an upper bound of $\sim 100 \mu \mathrm{M}$. That the binding of OA induces the same effect on the iron ligand field environment as the addition of substrate suggests comparable binding constraints. Since it is one aim of this study to investigate how substrate binding modulates the coordination in the active site, we have used OA to mimic the interactions between substrate and enzyme. In the following sections, the effects of (i) mutations

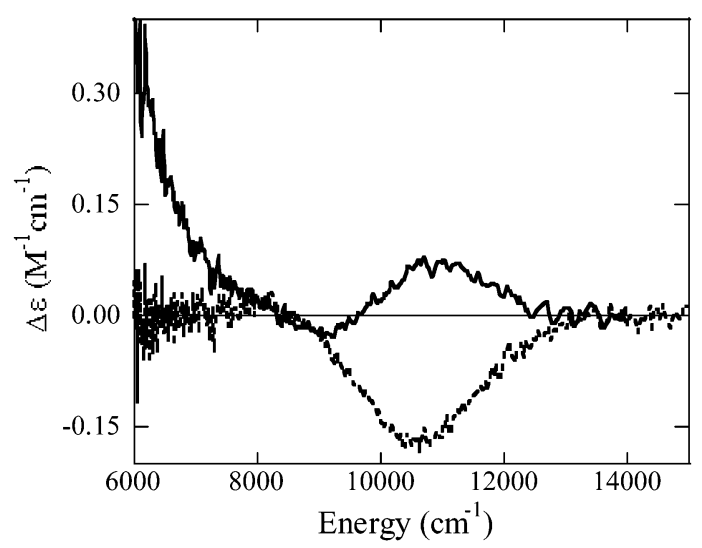

Figure 2: CD spectra at $5^{\circ} \mathrm{C}$ of wt sLO-1 in $50 \mathrm{mM}$ CHES, pD 9.0 (solid line) and wt sLO-1 plus 20 equiv of OA (dashed line). The spectrum of wt sLO-1 in the presence of $50 \%$ glycerol (28) is indistinguishable from the latter one and is, thus, omitted from the figure. Note that sucrose has no effect on the spectrum of the resting enzyme (28). Gaussian resolved spectra are appended in the Supporting Information.

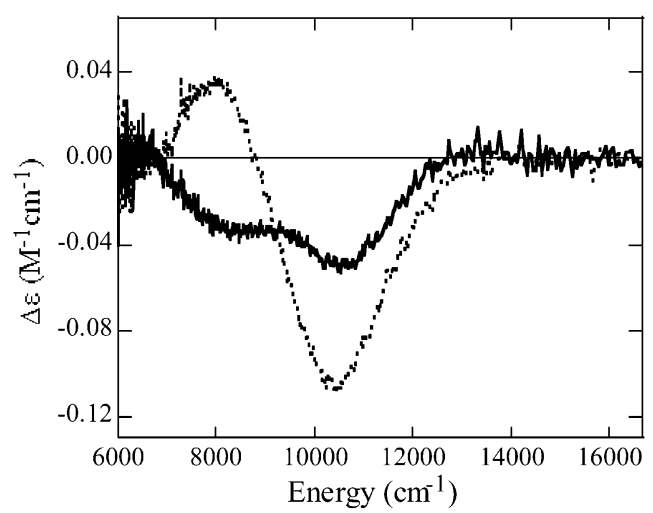

Figure 3: CD spectra at $5{ }^{\circ} \mathrm{C}$ of Q697E in $50 \mathrm{mM}$ CHES, pD 9.0 (solid line) and Q697E plus 20 equiv of OA (dashed line). Note that the spectra of Q697E in the presence of both 50\% glycerol and supersaturating sucrose are also very similar to that of the mutant in the presence of OA. Gaussian resolved spectra are appended in the Supporting Information.

in the second coordination sphere and (ii) binding of OA to these mutants are described in context of the observed coordination flexibility in sLO-1.

Q697E Spectroscopy. The near-IR CD spectrum of Q697E shows two negative features around 8000 and $10500 \mathrm{~cm}^{-1}$ (Figure 3). Gaussian resolution of the spectrum results in two bands at 8200 and $10500 \mathrm{~cm}^{-1}$, consistent with a $6 \mathrm{C}$ ferrous site, with distorted octahedral geometry (vide supra; note that the Gaussian fits were omitted from Figures 2 to 6 for clarity-Gaussian resolved spectra are attached in the Supporting Information). Similar to wt sLO-1, glycerol affects the ligand field of this mutant (data not shown). However, in contrast to the former, the $\mathrm{CD}$ spectrum of Q697E is also affected by sucrose. Both glassing agents lead to a $6 \mathrm{C}$ site different from that of the resting enzyme. Hence, it is currently not possible to study Q697E by MCD. The addition of $\mathrm{OA}$ also affects the $\mathrm{CD}$ transitions in a manner similar to both glycerol and sucrose (Figure 3 ). The spectrum resembles that of wt sLO- 1 in the presence of OA (Figure 2), with one positive band around $8000 \mathrm{~cm}^{-1}$ and one large negative feature at $\sim 10500 \mathrm{~cm}^{-1}$. Gaussian fitting resolves these features in two bands at 8100 and $10500 \mathrm{~cm}^{-1}$ (Table $1)$. 
Table 2: Kinetic Parameters for SLO and Second Sphere Mutants at $22{ }^{\circ} \mathrm{C}^{a}$

\begin{tabular}{lccc}
\hline & $k_{\text {cat }}$ & $K_{\mathrm{m}}$ & $k_{\text {cat }} / K_{\mathrm{m}}$ \\
\hline SLO & $287(5)$ & $15(1)$ & $19(1)$ \\
Q495E & $329(15)$ & $23(3)$ & $14(2)$ \\
Q495A & $68(2)$ & $12(1)$ & $5.7(0.5)$ \\
Q697N & $98(5)$ & $15(2)$ & $6.5(1)$ \\
Q697E & $45(2)$ & $21(2)$ & $2.1(0.2)$
\end{tabular}

${ }^{a} 100 \mathrm{mM}$ borate (pH 9.2), $k_{\mathrm{cat}}$ is in $\mathrm{s}^{-1}, K_{\mathrm{m}}$ is in $\mu \mathrm{M}$, and $k_{\mathrm{cat}} / K_{\mathrm{m}}$ is in $\mathrm{s}^{-1} \mu \mathrm{M}^{-1}$.

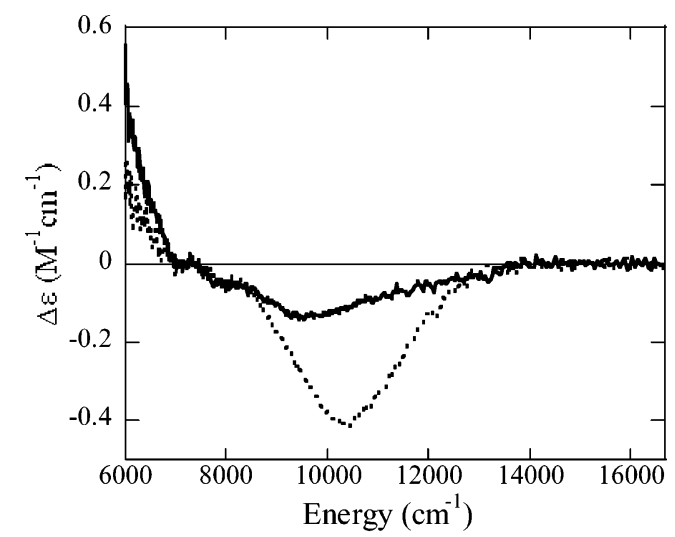

Figure 4: $\mathrm{CD}$ spectra at $5{ }^{\circ} \mathrm{C}$ of Q697N in $50 \mathrm{mM} \mathrm{CHES,} \mathrm{pD} 9.0$ (solid line) and Q697N plus 20 equiv of OA (dashed line). Note that the spectrum of Q697N in the presence of $50 \%$ glycerol is also very similar to that of the mutant in the presence of OA (not shown). Addition of sucrose has no effect on the CD spectrum of the resting enzyme. Gaussian resolved spectra are appended in the Supporting Information.

Significantly, in contrast to resting wt sLO-1, the Q697E mutant is $6 \mathrm{C}$ in the resting form and does not display coordination flexibility, remaining purely $6 \mathrm{C}$ upon substrate analogue (OA) binding. This result contradicts crystallography, which predicts a purely $5 \mathrm{C}$ geometry for this mutant (Figure 1) and reiterates the sensitivity of the active site geometry toward experimental conditions (28). Interestingly, while the addition of OA does not affect the coordination number, the ligand field is perturbed by this inhibitor, leading to a $6 \mathrm{C}$ geometry resembling that of the wt enzyme with bound OA (Figures 2 and 3). Since the substrate/ inhibitor bound $6 \mathrm{C}$ form is believed to be the catalytically relevant species, both wt and Q697E are expected to have similar reactivity because of their similar spectra when OA is added. The fact that their $k_{\text {cat }}$ values vary significantly (287 and $45 \mathrm{~s}^{-1}$, respectively, Table 2) (14) presents a clear indication that coordination flexibility of the sixth ligand is the primary physical property that affects the reactivity of Q697E, contributing approximately $5 \mathrm{~kJ} / \mathrm{mol}$ to the activation energy.

Q697N and Q495A Spectroscopy. The near-IR CD spectrum of resting Q697N is shown in Figure 4 and demonstrates the presence of a major, broad negative feature at $\sim 10000$ $\mathrm{cm}^{-1}$, a weaker negative shoulder at $\sim 8000 \mathrm{~cm}^{-1}$, and an intense positive band at $<6000 \mathrm{~cm}^{-1}$. Since no ligand field arrangement of a mononuclear ferrous site can have more than two $\mathrm{d} \rightarrow \mathrm{d}$ transitions, Q697N must exist as a mixture of two species, similar to the wt enzyme (28). Gaussian resolution of the spectrum in Figure 4 results in four peaks at $<6000,11225,8985$, and $10090 \mathrm{~cm}^{-1}$, where the first two transitions are ascribed to a square-planar 5C species

\begin{tabular}{ccc}
\hline Table 3: Percentage of Five-Coordinate Species Present in sLO-1 \\
\hline & $\% 5 \mathrm{C}$ & $\% 5 \mathrm{C}$ \\
enzyme & resting & $\begin{array}{c}\text { resting }+ \\
\text { oleate }\end{array}$ \\
\hline wild-type & 40 & 0 \\
Q697E & 0 & 0 \\
Q697N & 40 & 20 \\
Q495A & $40-45$ & 25 \\
Q495E & 40 & 0 \\
\hline
\end{tabular}

and the latter two transitions to a distorted octahedral $6 \mathrm{C}$ form. The relative contributions of the two species in the mixture were estimated by subtracting varying amounts of the $6 \mathrm{C}$ form from the spectrum in Figure 4 . The sixcoordinate species is slightly more abundant, contributing approximately $60 \%$ to the mixture (Table 3 ). This is similar to the wt enzyme, where the relative contributions of $6 \mathrm{C}$ and $5 \mathrm{C}$ species was estimated to 60 and $40 \%$, respectively (28). The presence of OA does alter the coordination environment of Q697N, but in contrast to the wt enzyme the mutant is not completely converted to the $6 \mathrm{C}$ form. Approximately $20 \%$ of unconverted 5C species remains (Figure 4). Not surprisingly, the ligand field of this mutant is also affected by the addition of glycerol to the same degree as OA (data not shown). The CD spectrum has been resolved into four bands at $<6000,11240,8630$, and $10160 \mathrm{~cm}^{-1}$, where again the first two transitions are ascribed to the $5 \mathrm{C}$ species and the latter two to the $6 \mathrm{C}$ form. The active site of Q697N is not affected by adding sucrose to supersaturation $(\sim 175 \mathrm{mg} / 100 \mu \mathrm{L})$, enabling the generation of an MCD sample. From the more intense transitions in the MCD spectrum (Figure S1, Supporting Information), the relative contributions of the two species after addition of OA, as estimated from the CD spectrum (Figure 4), were confirmed.

Figure 5a shows the near-IR CD spectrum of resting Q495A, which resembles that of the resting Q697N mutant (Figure 4), with a broad negative feature at $\sim 10000 \mathrm{~cm}^{-1}$ and a positive band at $<6000 \mathrm{~cm}^{-1}$. Because of the weak transitions, it was difficult to subject the $\mathrm{CD}$ spectrum to Gaussian fitting. Since sucrose does not affect the spectrum (data not shown), an MCD sample of resting Q495A could be prepared. The MCD spectrum at $7 \mathrm{~T}$ and $1.6 \mathrm{~K}$ (Figure $5 \mathrm{~b}$ ) was Gaussian resolved and indicates the presence of four bands at 5100, 11300,8500 , and $10500 \mathrm{~cm}^{-1}$. Again, the first two transitions are assigned to a $5 \mathrm{C}$ species, while the remaining two are characteristic of a $6 \mathrm{C}$ form (vide supra). The ratio of the two forms is similar to those of both wt and Q697N, with the 6C species slightly more abundant (Table 3 ). Addition of OA (or glycerol) affects the transitions in the $\mathrm{CD}$ and MCD spectra (Figure 5) leading to an increase in the $6 \mathrm{C}$ contribution. The resulting spectra resemble that of Q697N (Figures 4 and S1), with approximately 20-25\% of unconverted 5C species (Table 3).

Both Q697N and Q495A have similar kinetic properties (14) with their turnover numbers being approximately three times lower than that of wt sLO-1 but about two times higher than that of Q697E. Previous crystallographic data would have predicted a pseudo-5C geometry for Q495A and a purely 5C ligand arrangement for the Q697N mutant (Figure 1), suggesting that $\mathrm{Q} 697 \mathrm{~N}$ would be more reactive. In contrast, $\mathrm{CD}$ and MCD data show that both mutants have a 


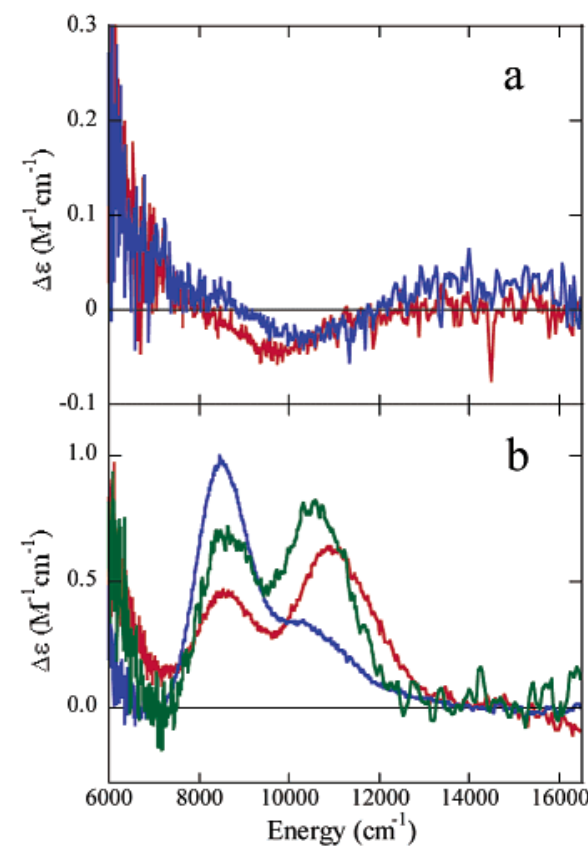

Figure 5: (a) CD spectra at $5^{\circ} \mathrm{C}$ of Q495A in $50 \mathrm{mM}$ CHES, pD 9.0 (red) and Q495A plus 20 equiv of OA (blue). The effect of adding $50 \%$ glycerol is again very similar to that observed upon addition of OA (not shown). Addition of sucrose has no effect on the $\mathrm{CD}$ spectrum of the resting enzyme. The $\mathrm{CD}$ intensities are too weak for Gaussian fitting. (b) MCD spectra at $7 \mathrm{~T}$ and $1.6 \mathrm{~K}$ of Q495A in $50 \mathrm{mM}$ CHES, pD 9.0 (red) and Q495A plus 20 equiv of OA (blue) in supersaturated sucrose solution $(\sim 175 \mathrm{mg} / \mu \mathrm{L})$. Also included is the MCD spectrum of resting wt sLO-1 (black) (28) for comparison. Gaussian resolved spectra are appended in the Supporting Information.

similar distribution between the $5 \mathrm{C}$ and the $6 \mathrm{C}$ species, and both are affected in a similar manner upon addition of OA. This spectroscopic observation is consistent with their similar kinetic properties (Table 2) and supports the hypothesis that the degree of flexibility in the first coordination sphere correlates with reactivity.

Q495E Spectroscopy. Figure 6a presents the near-IR CD spectra for the resting and resting $\mathrm{Q} 495 \mathrm{E}$ in the presence of OA. In the resting state, one major feature, below $6000 \mathrm{~cm}^{-1}$, is observed, with a broad tail in the higher energy region. The poor resolution of the CD spectrum does not allow Gaussian fitting, but from the intense feature at low energy it is evident that a substantial portion of the enzyme is $5 \mathrm{C}$. Addition of OA (and glycerol) alters the spectrum with only one negative band present at $\sim 10500 \mathrm{~cm}^{-1}$. The lack of a low energy transition indicates a complete conversion toward a purely $6 \mathrm{C}$ geometry (Table 3 ). Sucrose was again an appropriate glassing agent for the preparation of $\mathrm{MCD}$ samples. The relevant spectra are shown in Figure $6 \mathrm{~b}$. The spectrum of the resting enzyme is Gaussian resolved into four bands because of the presence of 5C $(<6000,12200$ $\left.\mathrm{cm}^{-1}\right)$ and $6 \mathrm{C}\left(8400,10800 \mathrm{~cm}^{-1}\right)$ species. Interestingly, the higher energy transitions are more resolved than in $\mathrm{wt}$ sLO-1 (because of a larger energy splitting of the ${ }^{5} \mathrm{E}_{\mathrm{g}}$ state in the $5 \mathrm{C}$ species), which leads to a distinctly different spectrum (Figure 6b). Hence, although both wt and Q495E are approximately $40 \% 5 \mathrm{C}$ in the resting state (Table 3 ) and convert to a purely $6 \mathrm{C}$ form upon $\mathrm{OA}$ addition, their ligand fields in the resting form are subject to different perturbations. From their crystal structures, it is seen that the main

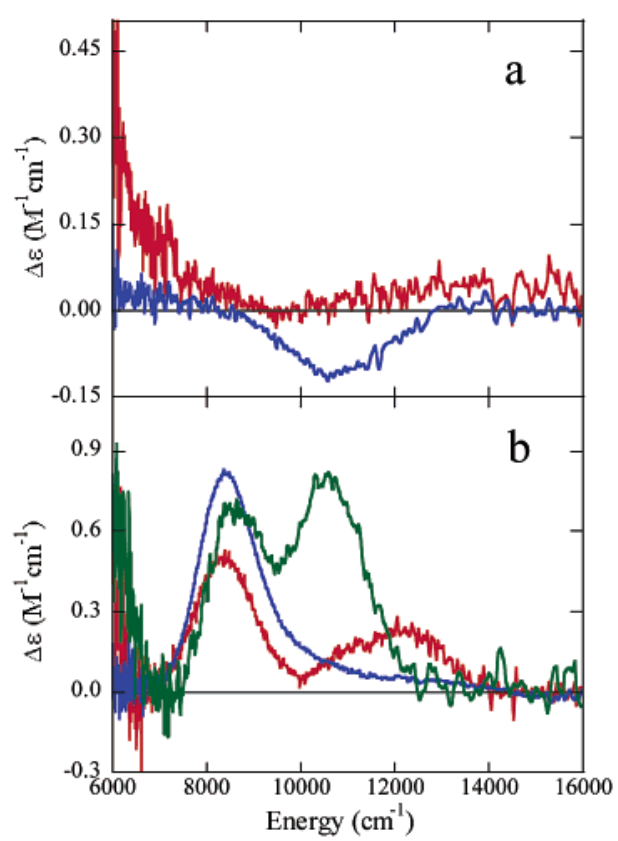

Figure 6: (a) CD spectra at $5{ }^{\circ} \mathrm{C}$ of Q495E in $50 \mathrm{mM}$ CHES, pD 9.0 (red) and Q495E plus 20 equiv of OA (blue). The effect of adding $50 \%$ glycerol is again very similar to that observed upon addition of OA (not shown). Addition of sucrose has no effect on the CD spectrum of the resting enzyme. Similar to the Q495A mutant (Figure 5) the CD intensities are too weak for Gaussian fitting. (b) MCD spectra at $7 \mathrm{~T}$ and $1.6 \mathrm{~K}$ of Q495E in $50 \mathrm{mM}$ CHES, pD 9.0 (red) and Q495E plus 20 equiv of OA (blue) in supersaturated sucrose solution $(\sim 175 \mathrm{mg} / \mu \mathrm{L})$. Also included is the MCD spectrum of resting wt sLO-1(black) (28) for comparison. Gaussian resolved spectra are appended in the Supporting Information.

difference between the two enzymes is the disruption of the hydrogen bond between the two second sphere residues, Gln697 and Glu495, in Q495E (Figure 1). In contrast to the other mutants in this study, Q495E and the wt enzyme exhibit an H-bond between His499 and position 495 (Figure 1). According to the crystal structures, His 499 is mobile in the wt structure (14). It was speculated that the interaction between His499 and Glu495, in Q495E, positions Glu495 in a similar orientation as Gln495 in the wt structure(14). The increased electrostatic repulsion in the active site may be the cause for the altered ligand field in the mutant as compared to wt sLO-1, at least in the resting state (Figure 6). However, in the inhibitor-bound form both enzymes have similar ligand field perturbations (Table 1), indicating a rearrangement of the $\mathrm{H}$-bond network that leads to similar $6 \mathrm{C}$ species. On the basis of the combined spectroscopic and structural data, it is evident that (i) the 6C site in Q495E strongly resembles that of the wt enzyme and (ii) that the coordination flexibility is not impaired Q495E.

\section{DISCUSSION}

Our groups have been interested in the correlation between the ligand coordination sphere of LO and its reactivity. We determined that the sixth ligand, Asn694 in sLO-1, is critical to the rate of the reaction by the observation that the stronger hisitidine ligation lowered the kinetic rate of both the 15$\mathrm{hLO}(33)$ and the N694H mutant of sLO-1 (36) $\left(k_{\mathrm{cat}}=280\right.$, 10 , and $6 \mathrm{~s}^{-1}$ for wt sLO-1, N694H, and 15-hLO, respectively). We have extended our studies to the second ligation 
sphere of sLO-1 and its potential role in catalysis (14). Specifically, we were interested in how the hydrogen bond network of Gln697 and Gln495 interacted with both the substrate cavity and the sixth ligand, Asn694, and we determined that the integrity of the hydrogen bond network did affect reactivity, suggesting a structural correlation. However, the crystallographically determined distance between Asn694 and the central metal ion (Figure 1) did not correlate with enzyme activity. This was an unexpected result because the interaction between the network and Asn694 would be the main conduit of a structure/function interaction. However, $\mathrm{CD} / \mathrm{MCD}$ spectroscopic data are more sensitive indicators of the first coordination sphere, as seen by the variation of the ligand arrangement depending on experimental conditions (28). While ligand bond lengths derived from crystallography may have to be viewed with caution, general structural features are represented reliably in the crystal structures; specifically, the hydrogen bond network connecting the first and second coordination spheres is welldefined in all known mammalian and plant LOs (24-27). In this study, the correlation of $\mathrm{Fe}^{\mathrm{II}}$ ligand field spectroscopic data with structural information has allowed us to investigate the roles individual interactions within the H-bond network play in the modulation of enzyme reactivity in LOs.

Crystallographic and spectroscopic data on wt SLO-1 and its four, second coordination sphere mutants are correlated to gain insight into the mechanism of $\mathrm{LO}$ catalyzed reactions. A crucial observation is that Q697E manifests a purely 6C species in the resting state (Figure 3); however, the wt enzyme displays a mixture of $5 \mathrm{C}$ and $6 \mathrm{C}$ species (Figure 2). This mutation also affects the $k_{\text {cat }}\left(\sim 40 \mathrm{~s}^{-1} ; 6\right.$-fold reduction, Table 2) more than any of the other second sphere mutations, while substrate binding is only slightly impaired (the $K_{\mathrm{m}}$ is an upper bound for the $K_{\mathrm{D}}$ for sLO-1 kinetics and is estimated for wt and Q697E to be 15 and $21 \mu \mathrm{M}$, respectively, Table 2) (14). Q697E is also the only mutation in the secondary coordination sphere, which leads to an inverse solvent isotope effect (SIE) and a shift of the $\mathrm{Fe}^{\mathrm{III}}$ ligand-to-metal chargetransfer transition to higher energy (14). These observations indicate a lower reduction potential of the iron site and correlate well with the reduced reactivity. However, the presence of a purely $6 \mathrm{C}$ species is in sharp contrast to the crystallographic results, which indicate a $5 \mathrm{C}$ geometry for resting Q697E: the distance between $\mathrm{Fe}^{\mathrm{II}}$ and Asn694 is 3.41 $\AA$ (14). As mentioned above, originally the octahedral coordination number for wt sLO-1 as determined from crystallography was also ill-defined $(24,25)$ until $\mathrm{CD}$ and MCD spectroscopy revealed the existence of a mixture of 5C and 6C species because of the weakly bound Asn694 ligand (28). Thus, while crystallography models imitate well the general protein environment, it can be less accurate with respect to specific ligand-metal bonding interactions. Therefore, in this study we use the crystal data to define the general orientation and interaction among residues leading to $\mathrm{H}$ bonding interactions, while we use the ligand field felt by the $\mathrm{Fe}^{\mathrm{II}}$ obtained through $\mathrm{CD}$ and MCD spectroscopies to probe the coordination environment of the $\mathrm{Fe}^{\mathrm{II}}$ center.

Despite the difficulty in determining the bond lengths in the primary coordination sphere, a comparison of the crystal structures for wt and Q697E still provides a possible explanation for the absence of coordination flexibility in the mutant. From the structures (Figure 1), it is apparent that the mutation introduced at Gln697 isolates Asn694 from the hydrogen bond network, which links the ligands Asn694 and His499 via Gln697 and Gln495 in wt sLO-1 (Figure 1) (14). In fact, all three of the H-bonds in the wt structure are absent in the Q697E mutant, and a new one is formed (Gln697His499). A recent theoretical study on wt sLO-1, using DFT, indicated that the tilted geometry of Asn694 (as imposed by the protein) results in a weak $\mathrm{Fe}^{\mathrm{II}}-\mathrm{Asn}$ bond (18). The constraint that maintains this tilted conformation can be modulated via the H-bond network that links Asn694 to the second coordination sphere. Thus, complete removal of this H-bond interaction would allow Asn694 to rearrange and form a stronger bond to $\mathrm{Fe}^{\mathrm{II}}$, leading to a purely $6 \mathrm{C}$ ligand arrangement.

The structures of Q697N and Q495A demonstrate the presence of only one H-bond (residues 697-694) of the original three in the wt enzyme. Nevertheless, this is the H-bond that interacts with the flexible Asn694 and maintains the $5 \mathrm{C} / 6 \mathrm{C}$ mixture. This is supported by the $\mathrm{CD}$ and $\mathrm{MCD}$ spectroscopy, which reveal that in solution both mutants are mixtures of $5 \mathrm{C}$ and $6 \mathrm{C}$ species (40:60 ratio) (Figures 4 and $5)$, similar to the wt enzyme. Again, this is not reflected in their crystal structures, with a purely $5 \mathrm{C}$ ligation for Q697N, and a pseudo-5C environment for Q495A, with $\mathrm{Fe}^{\mathrm{II}}-\mathrm{Asn} 694$ distances of 3.51 and $2.93 \AA$, respectively (14). Furthermore, the mutations do not lead to greatly altered ligand fields (Table 1), which indicate that in the resting state the catalytic ability of the mutants should not be significantly perturbed relative to the wt enzyme. Nonetheless, their reaction rates are reduced (Q697N $k_{\text {cat }}=68 \mathrm{~s}^{-1}$ and Q495A $k_{\text {cat }}=98 \mathrm{~s}^{-1}$, Table 2), which correlates well with their spectroscopic differences in metal ligation in the OA-bound form and suggests that these differences are catalytically relevant (vide infra).

In contrast to the other three mutants, which only have one H-bond in the network, Q495E has two H-bond interactions between the first and the second sphere residues (Figure 1), Gln697-Asn694 and Glu495-His499 (Figure 1). From the crystal structure, the $\mathrm{Fe}^{\mathrm{II}}-\mathrm{Asn} 694$ distance (2.89 $\AA)$ is almost identical to that of wt sLO-1 $(2.87 \AA)$ and suggests a pseudo-5C environment (14). However, CD and MCD spectroscopy again reveal that in solution this mutant consists of a mixture of $5 \mathrm{C}$ and $6 \mathrm{C}$ species (Figure 6). The percentage of $5 \mathrm{C}$ species in the resting form is approximately $40 \%$ (Table 3), similar to the wt enzyme and the Q697N and Q495A mutants. The ligand field of this $5 \mathrm{C}$ species is different from the other sLOs (Table 1), with $\Delta \mathrm{E}_{\mathrm{g}}$ being unusually large $\left(>6000 \mathrm{~cm}^{-1}\right)$. Apart from the wt enzyme, $\mathrm{Q} 495 \mathrm{E}$ is the only mutant where the second coordination sphere residue at position 495 is $\mathrm{H}$-bonded to the equatorial ligand His499 (Figure 1). The replacement of a glutamine by a glutamate in position 495 increases the electrostatic interaction with His499, leading to a stronger ligand field from His499. This effect is also visible in the $6 \mathrm{C}$ form, although not as strong as the $5 \mathrm{C}$ (Table 1) since the $\mathrm{Fe}^{\mathrm{II}}-$ Asn694 bond partially compensates the charge donation from His499. It is interesting to note that Q697E, where the second coordination sphere residue in position 697 is H-bonded to His499, also has a stronger ligand field (Table 1). Hence, interactions between the second coordination sphere and the equatorial ligand His499 do affect the first coordination sphere; however, the implications on catalysis are minimal 
since the Q495E mutant is slightly more reactive than the wt enzyme ( $k_{\text {cat }}=329$ and $287 \mathrm{~s}^{-1}$, respectively), while Q697E is significantly less reactive $\left(k_{\mathrm{cat}}=45 \mathrm{~s}^{-1}\right.$, Table 2$)$ (14).

In the least active mutant (Q697E), the axial Asn694 is uncoupled from the $\mathrm{H}$-bond network, and thus, its active site remains purely $6 \mathrm{C}$. The other three mutants resemble $\mathrm{wt}$ sLO-1 in the resting state and maintain an $\mathrm{H}$-bond between residues 697 and Asn694. Nevertheless, this model does not explain the difference in their enzymatic rates. To understand why two of these mutants (Q697N and Q495A) have reduced reactivity as compared to the wt enzyme and Q495E, we need to also consider the effect of a substrate analogue (OA) binding to the enzyme. Figure 2 shows that addition of OA to the wt enzyme triggers the rearrangement of the $\mathrm{H}$-bond network, leading to the disappearance of the $5 \mathrm{C}$ species. The ligand field of the $6 \mathrm{C}$ form is not perturbed upon OA binding (Table 1), indicating that the inhibitor does not directly interact with the metal center once the $6 \mathrm{C}$ species is formed. In Q697E, the effect of OA binding is less dramatic (Figure 3) because of the uncoupling of Asn694 from the H-bond network. The ligand field is not greatly perturbed (i.e., the ligand field energies do not change) by the interaction with the inhibitor (Table 1); however, some conformational change does occur in the vicinity of the active site, based on the change of sign of the CD spectrum for the lower energy transition (Figure 3). A similar observation was seen for the binuclear methane monooxygenase, where the addition of the inhibitor, tetrachloroethylene, in the presence of component B (a bound low-molecular weight protein that allows catalysis), induces a sign change in one of the $d-d$ transitions (45). The fact that OA does not perturb the ligand fields of the 6C species in both wt and Q697E further supports the role of coordination flexibility of Asn694 as the main modulator of enzyme activity. In the proposed sLO mechanism, the active form of the enzyme is in the pseudo5C ferric state (vide supra). The stronger Fe-Asn694 bond in the Q697E mutant could lead to a stabilization of the ferric oxidation state, lowering of the reduction potential, and thus, decreasing reactivity. This hypothesis is consistent with the observed shift of the characteristic His $-\mathrm{Fe}^{\mathrm{III}}$ ligand-to-metal charge-transfer transition at $416 \mathrm{~nm}$, which was interpreted in terms of a decrease in the reduction potential of the iron (14).

As mentioned above, the CD/MCD spectra of Q697N and Q495A resemble the wt enzyme in their resting state with a mixture of $5 \mathrm{C}$ and $6 \mathrm{C}$ sites. However, in contrast to $\mathrm{wt}$ sLO-1, the addition of OA to these two mutants alters this ratio only moderately in favor of the $6 \mathrm{C}$ species (Table 3 ), and the ligand fields of both the $5 \mathrm{C}$ and the $6 \mathrm{C}$ species are not greatly affected (Table 1 ). The diminished effect of OA binding to the first coordination sphere of the two mutants may be due to the fact that the second sphere residue in position 495 is completely uncoupled from the hydrogen bond network (Figure 1). In the recently published crystal structure of the product-bound (purple) ferric sLO-3 species, it has been shown that the major structural change upon product binding is a reorganization of Gln495 (Gln514 in sLO-3), which leads to the disruption of the H-bond network and the formation of a $6 \mathrm{C}$ active site (23). In the absence of crystallographic data for the two mutants with bound substrate or OA, it is difficult to assess factors that may contribute to the incomplete conversion. However, the crystal structures of their resting ferrous states (Figure 1) show (i) that Gln495 is mobile in Q697N and (ii) that water molecules fill the space around Ala495 in Q495A, indicating that interactions between the enzyme and the inhibitor/substrate in the region around residue 495 may not be as rigid as in wt sLO-1. A model of sLO-1 with linoleate docked into the substrate binding cavity indicates that Q495 may be crucial for the precise positioning of the substrate near the catalytic center (14).

$\mathrm{Q} 495 \mathrm{E}$ is the only mutant investigated where the addition of OA leads to a complete conversion to the $6 \mathrm{C}$ form (Table 3 ), while the ligand field is not greatly perturbed (Table 1). The observation that the kinetic efficiency of this mutant is not impaired stresses the importance of coordination flexibility in the regulation of enzyme activity. It is important to note that the H-bond network of Q495E is also lacking the interaction between residue 495 and 697, as seen in Q697N and Q495A. However, the conversion to 6C and the activity are not affected. Interestingly, Q495E does have a H-bond between Glu495 and His499, which is not present in the other three mutants (Figure 1) but is seen in the wt enzyme, suggesting a role in activity.

It should be emphasized that while the coordination flexibility contribution to catalysis specifically involves the redox $/ \mathrm{p} K_{\mathrm{a}}$ of the $\mathrm{Fe}^{\mathrm{II}} / \mathrm{Fe}^{\mathrm{III}}$ couple, we have shown previously that the coordination flexibility of the ferrous species with substrate binding directly relates to the ferrous/ferric state reactivity. The coordination flexibility was originally observed with substrate or substrate-analogue binding to the $\mathrm{Fe}^{\mathrm{II}}$ site of wt sLO-1 but not observed for mammalian or N694H sLO-1, and thus, was associated with the Asn ligand and reproduced in DFT calculations. This correlates with the ligation differences observed with EPR of the $\mathrm{Fe}^{\mathrm{III}}$ forms of these enzymes (axial in wt sLO-1 in the presence or absence of bound substrate analogue (Zhou, Holman, and Solomon, unpublished experiment), rhombic in mammalian, and N694H sLO-1) and their differences in reactivity.

In conclusion, the data presented herein combine MCD/ CD spectroscopy with X-ray crystallography to investigate the role of the sixth ligand in SLO-1 (Asn694). MCD/CD is sensitive to bonding interactions within the coordination sphere and has allowed us to correlate the iron coordination with the enzymatic activity of the mutations. The data presented above demonstrate that coordination flexibility at Asn694 is crucial for the modulation of enzyme reactivity in SLO-1; the higher the flexibility the more reactive the enzyme. In other words, we do observe for the resting enzyme that the iron environment does directly relate to activity, with the mutations that have the ability to form a $5 \mathrm{C} / 6 \mathrm{C}$ mixture being more active. The model that emerges confirms that the hydrogen bond network between Asn694, Gln697, Gln495, and His499 (Figure 1) connects the substrate binding site to the iron coordination environment, and in particular, stresses the importance of the H-bond between the weak Asn694 ligand and Gln697 in modulating coordination flexibility and thus enzyme activity. When the flexible residue Asn694 itself is uncoupled from the H-bond network, the active site is purely $6 \mathrm{C}$, independent of the addition of substrate/inhibitor or small organic molecules (Q697E). When the second sphere residue in position 495 is uncoupled from the H-bond network (Q697N, Q495A), 
the flexibility is restricted with $\sim 20 \%$ of the active sites still being in $5 \mathrm{C}$ form after the addition of inhibitor, and by analogy, substrate (vide supra). The reactivity of these two mutants is therefore reduced, indicating that improper positioning of the substrate may account for the partial conversion to pure $6 \mathrm{C}$. Finally, the mutation that maintains both the 697-694 and the 495-499 interactions (Q495E) retains the Asn694 flexibility and activity comparable to wt enzyme.

\section{ACKNOWLEDGMENT}

The authors would like to thank Dr. Nataša Mitić for helpful discussions and technical assistance.

\section{SUPPORTING INFORMATION AVAILABLE}

Gaussian resolved spectra for wt sLO-1 and its second coordination sphere mutants. This material is available free of charge via the Internet at http://pubs.acs.org.

\section{REFERENCES}

1. Siedow, J. N. (1991) Annu. Rev. Plant Physiol. Plant Mol. Biol $42,145-188$

2. Yamamoto, S. (1992) Biochim. Biophys. Acta 1128, 117-131.

3. Solomon, E. I., Zhou, J., Neese, F., and Pavel, E. G. (1997) Chem. Biol. 4, 795-808.

4. Samuelsson, B., Dahlen, S.-E., Lindgren, J. A., Rouzer, C. A., and Serhan, C. N. (1987) Science 237, 1171-1176.

5. Sigal, E. (1991) Am. J. Physiol. 260, L13-L28.

6. van Leyen, K., Duvoisin, R. M., Engelhardt, H., and Wiedmann, M. (1998) Nature 395, 392-395.

7. Kuhn, H., and Thiele, B. J. (1999) FEBS Lett. 449, 7-11.

8. Honn, K. V., Tang, D. G., Gao, X., Butovich, I. A., Liu, B., Timar, J., and Hagmann, W. (1994) Cancer Metastasis Rev. 13, 365396.

9. Axelrod, B., Cheesbrough, T. M., and Laakso, S. (1981) Methods Enzymol. 71, 441-451.

10. Cheesbrough, T. M., and Axelrod, B. (1983) Biochemistry 22, 3837-3840.

11. Feiters, M. C., Aasa, R., Malmstroem, B. G., S., S., Veldink, G. A., and Vliegenhart, J. F. G. (1985) Biochim. Biophys. Acta 831, $302-305$.

12. Veldink, G. A., and Vliegenhart, J. F. G. (1984) Adv. Inorg. Biochem. 6, 139-161.

13. Scarrow, R. C., Trimitsis, M. G., Buck, C. P., Grove, G. N., Cowling, R. A., and Nelson, M. J. (1994) Biochemistry 33, 15023-15035.

14. Tomchick, D. R., Phan, P., Cymborowski, M., Minor, W., and Holman, T. R. (2001) Biochemistry 40, 7509-7517.

15. Glickman, M. H., and Klinman, J. P. (1996) Biochemistry 35, 12882-12892.

16. Nelson, M. J., Seitz, S. P., and Cowling, R. A. (1990) Biochemistry $29,6897-6903$.

17. Glickman, M. H., and Klinman, J. P. (1995) Biochemistry 34 14077-14092.

18. Lehnert, N., and Solomon, E. I. (2002) J. Biol. Inorg. Chem. 8, 294-305.
19. Nelson, M. J., and Cowling, R. A. (1990) J. Am. Chem. Soc. 112, $2820-2821$.

20. Nelson, M. J., Cowling, R. A., and Seitz, S. P. (1990) Biochemistry 29, 6897-6903.

21. Chamultriat, W., and Mason, R. P. (1989) J. Biol. Chem. 264, 20968-20973.

22. Egmond, M. E., Fasella, P. M., Veldink, G. A., Vliegenhart, J. F. G., and Boldingh, J. (1977) Eur. J. Biochem. 76, 469-479.

23. Skrzypczak-Jankun, E., Bross, R. A., Carroll, R. T., Dunham, W. R., and Funk, J., M. O. (2001) J. Am. Chem. Soc. 123, 1081410820.

24. Boyington, J. C., Gaffney, B. J., and Amzel, L. M. (1993) Science $260,1482-1486$

25. Minor, W., Steczko, J., Stec, B., Otwinowski, Z., Bolin, J. T., Walter, R., and Axelrod, B. (1996) Biochemistry 35, 1068710701.

26. Skrzypczak-Jankun, E., Amzel, L. M., Kroa, B. A., and Funk, J. M. O. (1997) Proteins Struct. Funct. Genet. 29, 15-31.

27. Gillmor, S. A., Villasenor, A., Fletterick, R., Sigal, E., and Browner, M. (1997) Nat. Struct. Biol. 4, 1003-1009.

28. Pavlosky, M. A., Zhang, Y., Westre, T. E., Gan, Q.-F., Pavel, E. G., Campochiaro, C., Hedman, B., Hodgson, K. O., and Solomon, E. I. (1995) J. Am. Chem. Soc. 117, 4316-4327.

29. Slappendel, S., Veldink, G. A., Vliegenhardt, J. F. G., Aasa, R., and Malmström, B. G. (1980) Biochim. Biophys. Acta 642, 3039.

30. Slappendel, S., Veldink, G. A., Vliegenthart, J. F. G., Aasa, R., and Malmström, B. G. (1983) Biochim. Biophys. Acta 747, 32.

31. Zhang, Y., Gebhard, M. S., and Solomon, E. I. (1991) J. Am. Chem. Soc. 113, 5162-5175.

32. Gaffney, B. J., Mavrophilipos, D. V., and Doctor, K. S. (1993) Biophys. J. 64, 773-783.

33. Zhang, Y., Gan, Q.-F., Pavel, E. G., Sigal, E., and Solomon, E. I (1995) J. Am. Chem. Soc. 117, 7422-7427.

34. Gardner, K. A., and Mayer, J. M. (1995) Science 269, 18491851.

35. Sloane, D. L., Leung, R., Barnett, J., Craik, C. S., and Sigal, E. (1995) Protein Eng. 8, 275-282.

36. Holman, T., Zhou, J., and Solomon, E. (1998) J. Am. Chem. Soc $120,12564-12572$.

37. Ballhausen, C. J. (1962) Introduction to Ligand Field Theory, MacGraw-Hill, New York.

38. Figgis, B. N. (1966) Introduction to Ligand Fields, Interscience, New York.

39. Solomon, E. I., Pavel, E. G., Loeb, K. E., and Campochiaro, C. (1995) Coord. Chem. Rev. 144, 369-460.

40. Solomon, E. I., Brunold, T. C., Davis, M. I., Kemsley, J. N., Lee, S. K., Lehnert, N., Neese, F., Skulan, A. J., Yang, Y. S., and Zhou, J. (2000) Chem. Rev. 100, 235-349.

41. Companion, A. L., and Komarynsky, M. A. (1964) J. Chem. Educ $41,257-262$.

42. Pavel, E. G., Kitjima, N., and Solomon, E. I. (1998) J. Am. Chem Soc. 120, 3949-3962.

43. Van der Heijdt, L. M., Feiter, M. C., Navaratnam, S., Nolng, H. F., Hermes, C., Veldink, G. A., and Vliegenhart, J. F. G. (1992) Eur. J. Biochem. 207, 793-802.

44. Mogul, R., Johansen, E., and Holman, T. R. (2000) Biochemistry 39, 4801-4807.

45. Pulver, S. C., Froland, W. A., Lipscomb, J. D., and Solomon, E. I. (1997) J. Am. Chem. Soc. 119, 387-395.

BI027380G 